BULL. AUSTRAL. MATH. SOC.

$20 F 05,20 F 25$

VOL. 9 (1973), 473-474.

\title{
Fibonacci sequences and
}

\section{group theory}

\section{A.M. Brunner}

Three topics are studied in the thesis.

In the first a sequence of elements of a group, called a Fibonacci sequence, is considered. Given an arbitrary group $G$ and two of its elements $a, b$ a Fibonacci sequence on $G$ with initial values $a$ and $b$ is the sequence: $a, b, b a, b a b, b a b^{2} a, \ldots$, where each three consecutive terms are of the form $u, v, v u$. For a particular group, and initial values, our main interest is in whether the Fibonacci sequence is periodic or not; if it is, then we wish to know the least positive period, called the length of the sequence. We obtain information, mostly of a combinatorial nature, about the freest two generator group to have a Fibonacci sequence of a given length - mainly through the study of some of its factor groups. Also included are some examples in which the lengths of Fibonacei- sequences are computed directly from the presentation of a group. Fibonacci sequences on non-abelian groups have previously been studied in [4].

The second topic is $T$-systems of groups. These were first defined in [2], and for an n-generator group are certain equivalence classes of the generating $n$-tuples of the group. Fibonacci sequences are used to determine the T-systems of several well known finite groups. A two generator group with an abelian normal subgroup such that the quotient group with respect to this subgroup is infinite cyclic, is shown to have one T-system of generating pairs. Our main result on $T$-systems is to show that the group $\operatorname{BP}\left(a, b ; b^{-1} a^{2} b=a^{3}\right)$ has an infinite number of

Received 28 August 1973. Thesis submitted to the Australian National University, April 1973. Degree approved, September 1973. Supervisors: Dr R.M. Bryant, Professor B.H. Neumann. 
T-systems of generating pairs. A presentation is associated with a representative generating pair in each of these T-systems. For each positive integer $n$ this presentation is

$$
\left(x, y ; x^{-1}[x, y]^{2},\left[x, x^{y^{n}}\right]\right) \text {. }
$$

Similar work, in which the T-systems of the trefoil knot group have been computed, has recently been done in [1].

The third topic is an isomorphism problem for the class of two generator one relator groups which are an extension of a free group of finite rank by a free group of rank one. The problem is that of determining, from their one relator presentations, whether or not two groups in this class are isomorphic. It is found to be sufficient to consider certain "standard" presentations. Our results then provide criteria which make it possible to distinguish most of these groups, modulo their second derived groups, by means of these standard presentations. For the class of groups which are an extension of a free group of rank two by a free group of rank one a complete solution to the problem is given which generalizes results in [3].

\section{References}

[1] M.J. Dunwoody and A. Pietrowski, "Presentations of the trefoil groups", Canad. Math. Bulz. (to appear).

[2] Bernhard H. Neumann und Hanna Neumann, "Zwei Klassen charakteristischer Untergruppen und ihre Faktorgruppen", Math. Nachr. 4 (1951), 106-125.

[3] Elvira Strasser Rapaport, "Note on Nielsen transformations", Proc. Amer. Math. Soc. 10 (1959), 228-235.

[4] Friedrich Roesler, "Fibonacci-Folgen in endlichen Gruppen", Math. Ann. 182 (1969), 154-160. 